\title{
On the holomorph of finite semisimple groups
}

\author{
Russell D. Blyth and Francesco Fumagalli* \\ Communicated by Timothy C. Burness
}

\begin{abstract}
Given a finite nonabelian semisimple group $G$, we describe those groups that have the same holomorph as $G$, that is, those regular subgroups $N \simeq G$ of $S(G)$, the group of permutations on the set $G$, such that $N_{S(G)}(N)=N_{S(G)}(\rho(G))$, where $\rho$ is the right regular representation of $G$.
\end{abstract}

\section{Introduction}

Let $G$ be any finite group, and let $S(G)$ be the symmetric group on the set of elements of $G$. We denote by $\rho: G \rightarrow S(G)$ and $\lambda: G \rightarrow S(G)$ respectively the right and the left regular representations of $G$. The normalizer

$$
\operatorname{Hol}(G)=N_{S(G)}(\rho(G))
$$

is the holomorph of $G$, and it is isomorphic to the natural extension of $G$ by its automorphism group $\operatorname{Aut}(G)$. It is well known that $\operatorname{Hol}(G)=N_{S(G)}(\lambda(G))$.

In [10], the multiple holomorph of $G$ has been defined as

$$
\operatorname{NHol}(G)=N_{S(G)}(\operatorname{Hol}(G)),
$$

and it is proved that the quotient group

$$
T(G)=\operatorname{NHol}(G) / \operatorname{Hol}(G)
$$

acts regularly by conjugation on the set of the regular subgroups $N$ of $S(G)$ that are isomorphic to $G$ and have the same holomorph as $G$, that is, $T(G)$ acts regularly on the set

$$
\mathscr{H}(G)=\left\{H \leq S(G) \mid H \text { is regular, } H \simeq G, N_{S(G)}(H)=\operatorname{Hol}(G)\right\} .
$$

There has been some attention both in the distant past [11] and quite recently [3-5, 9] on the problem of determining, for $G$ in a given class of groups, those groups that have the same holomorph as $G$ and, in particular, the set $\mathscr{H}(G)$ and 
the structure of the group $T(G)$. Recently, in [5], the authors attack this problem when $G$ is a perfect group, obtaining complete results for centerless groups [5, Theorem 7.7]. However, they leave open some interesting questions when the center is nontrivial.

The aim of this paper is to completely resolve the case when $G$ is a finite semisimple group.

One of the main obstacles for describing the holomorph of a finite semisimple group (see [5, Remark 7.12] and also [2, ADV - 4B]) was to completely classify those finite nonabelian simple groups that admit automorphisms acting like inversion on their Schur multiplier. In Proposition 2, we produce a complete analysis, whose proof depends on the Classification of Finite Simple Groups. It turns out that there is a small list $\mathscr{L}$ (see after Proposition 2 for its definition) of related quasisimple groups having automorphisms inverting their center. Our main result can be therefore stated as follows.

Theorem. Let $G$ be a finite nonabelian semisimple group, and let

$$
G=A_{1} A_{2} \ldots A_{n}
$$

be its unique central decomposition as a product of $\operatorname{Aut}(G)$-indecomposable factors. Assume that the number of factors $A_{i}$ of $G$ having components in $\mathscr{L}$ is exactly l for some $0 \leq l \leq n$. Then $T(G)$ is an elementary abelian group of order $2^{h}$ for some $h$ with $\min \{n-l+1, n\} \leq h \leq n$, acting regularly on $\mathscr{H}(G)$. Moreover, if the centers of the factors $A_{i}$ are all amalgamated, then $|\mathcal{H}(G)|=2^{m}$, and $T(G)$ is elementary abelian of order $2^{m}$, where $m=\min \{n, n-l+1\}$, and therefore $|\mathcal{H}(G)|=2^{m}$.

\section{Semisimple groups}

To establish the notation, note that we write permutations as exponents, and denote compositions of maps by juxtaposition. We compose maps left-to-right. We consider the right and the left regular "representations" of $G$, defined by

$$
\begin{array}{rlrl}
\rho: G & \rightarrow S(G) & \lambda: G & \rightarrow S(G), \\
g & \mapsto(x \mapsto x g), \quad g & \mapsto(x \mapsto g x),
\end{array}
$$

Remark 1. Since composition of maps is left-to-right, with our definition, the map $\lambda$ is an antihomomorphism, not a homomorphism, from $G$ to $S(G)$. We have chosen this definition over the standard one (where $\lambda(g)$ maps $x \in G$ to $g^{-1} x$ ) for the same reasons as in [5]. 
The first proposition recalls some basic facts (see [4, Proposition 2.4]). The proof is left to the reader.

Proposition 1. Let $G$ be any group, and let inv be the inversion map on $G$ defined by $\operatorname{inv}(g)=g^{-1}$ for every $g \in G$. The following hold.

(1) $C_{S(G)}(\rho(G))=\lambda(G)$ and $C_{S(G)}(\lambda(G))=\rho(G)$.

(2) $N_{S(G)}(\rho(G))=\operatorname{Aut}(G) \ltimes \rho(G)=\operatorname{Aut}(G) \ltimes \lambda(G)=N_{S(G)}(\lambda(G))$.

(3) $\rho(g)^{\mathrm{inv}}=\lambda\left(g^{-1}\right)$ and $\lambda(g)^{\mathrm{inv}}=\rho\left(g^{-1}\right)$ for every $g \in G$. In particular, inv conjugates $\rho(G)$ to $\lambda(G)$ (and vice versa), and it centralizes $\operatorname{Aut}(G)$; therefore, inv normalizes $N_{S(G)}(\rho(G))$, that is, inv $\in \operatorname{NHol}(G)$.

Recall that a quasisimple group is a perfect group $X$ such that $X / Z(X)$ is simple, and that a semisimple group is a central product of quasisimple groups, that is, a group $X=X_{1} X_{2} \ldots X_{t}$ with each $X_{i}$ quasisimple and such that $\left[X_{i}, X_{j}\right]=1$ for every $i \neq j$. The quasisimple normal subgroups $X_{i}$ of $X$ are called the components of $X$. Note in particular that semisimple groups are perfect.

Every finite semisimple group admits a unique decomposition as a central product of characteristic subgroups, which we call a central decomposition as in the theorem. We also remind the reader that a group $H$ is said to be centrally indecomposable as an $\operatorname{Aut}(H)$-group if it cannot be expressed as the central product of two proper characteristic subgroups.

Lemma 1. Let $G$ be a finite semisimple group. Then $G$ is a central product of perfect subgroups which are centrally indecomposable as $\operatorname{Aut}(G)$-subgroups,

$$
G=A_{1} A_{2} \ldots A_{n}
$$

Moreover, the integer $n$ and the subgroups $A_{i}$ (for $\left.i=1,2, \ldots, n\right)$ are uniquely determined (up to permutation).

Proof. Consider the Remak-Krull-Schmidt decomposition of $\operatorname{Inn}(G) \simeq G / Z(G)$ as an $\operatorname{Aut}(G)$-group, and let this be

$$
\frac{G}{Z(G)}=\frac{M_{1}}{Z(G)} \times \frac{M_{2}}{Z(G)} \times \cdots \times \frac{M_{n}}{Z(G)} .
$$

Since $G$ is perfect, each $M_{i} / Z(G)$ is perfect. In particular, each $M_{i}$ is equal to $M_{i}^{\prime} Z(G)$, where $M_{i}^{\prime}$ is perfect. Now, for every $j \neq i$, we have that

$$
\left[M_{j}, M_{i}^{\prime}\right]=\left[M_{j}, M_{i}\right] \leq Z(G),
$$


and thus $M_{j}$ induces by conjugation a central automorphism on $M_{i}^{\prime}$. Since perfect groups have no nontrivial central automorphisms (see [8] or [5, Lemma 7.1]), we have $\left[M_{i}, M_{j}\right]=1$ for every $j \neq i$. In particular, equation (2.1) and the fact that $G$ is perfect imply the following central factorization of $G$ :

$$
G=A_{1} A_{2} \ldots A_{n},
$$

where $A_{i}=M_{i}^{\prime}$ for each $i=1,2, \ldots, n$. Note that the $A_{i}$ are perfect $\operatorname{Aut}(G)$ subgroups, which are indecomposable as $\operatorname{Aut}(G)$-subgroups. Finally, the uniqueness of the factorization (2.1) (see [12, Theorem 3.3.8]) and, again, the fact that perfect groups have no nontrivial central automorphisms imply that the central product decomposition (2.2) is also unique.

We make use of the same notation as [5]. In particular, we define the following subsets of subgroups of $S(G)$ :

$$
\begin{aligned}
\mathscr{H}(G) & =\left\{H \leq S(G) \mid H \text { is regular, } H \simeq G, N_{S(G)}(H)=\operatorname{Hol}(G)\right\}, \\
\mathcal{L}(G) & =\left\{H \leq S(G) \mid H \text { is regular, } N_{S(G)}(H)=\operatorname{Hol}(G)\right\}, \\
\mathcal{J}(G) & =\left\{H \leq S(G) \mid H \text { is regular, } N_{S(G)}(H) \geq \operatorname{Hol}(G)\right\} .
\end{aligned}
$$

Note that $\mathscr{H}(G) \subseteq \mathcal{d}(G) \subseteq \mathcal{A}(G)$.

From now on, we assume that $G$ is a finite semisimple group, and we write $G$ as a central product of indecomposable $\operatorname{Aut}(G)$-subgroups, in a unique way (by Lemma 1) as $G=A_{1} A_{2} \ldots A_{n}$. Note that, by Proposition 1 (1), we have that $\left[\rho\left(A_{i}\right), \lambda\left(A_{j}\right)\right]=1$ for every $i \neq j$.

Fix $I$ to be the set $\{1,2, \ldots, n\}$. For each subset $J$ of $I$, we denote the central product $\prod_{j \in J} A_{j}$ by $A_{J}$. Then, for each subset $J$ of $I$, we may define the subgroup $G_{J}$ of $S(G)$ to be $G_{J}=\rho\left(A_{J}\right) \lambda\left(A_{J^{c}}\right)$, where $J^{c}=I \backslash J$. Note that $G_{I}=\rho(G)$ and $G_{\varnothing}=\lambda(G)$.

Lemma 2. Each $G_{J}$ is a subgroup of $S(G)$ that lies in $\operatorname{Hol}(G)$.

Proof. Since $[\rho(G), \lambda(G)]=1$, for every $x_{J}, y_{J} \in A_{J}, x_{J^{c}}, y_{J^{c}} \in A_{J^{c}}$, it follows that

$$
\begin{aligned}
\rho\left(x_{J}\right) \lambda\left(x_{J^{c}}\right)\left(\rho\left(y_{J}\right) \lambda\left(y_{J^{c}}\right)\right)^{-1} & =\rho\left(x_{J}\right) \rho\left(y_{J}\right)^{-1} \lambda\left(x_{J^{c}}\right) \lambda\left(y_{J^{c}}\right)^{-1} \\
& =\rho\left(x_{J} y_{J}^{-1}\right) \lambda\left(y_{J^{c}}^{-1} x_{J^{c}}\right),
\end{aligned}
$$

which lies in $G_{J}$.

Moreover, since $\rho\left(A_{J}\right) \leq \rho(G)$ and $\lambda\left(A_{J^{c}}\right) \leq \lambda(G)$, we have

$$
G_{J}=\rho\left(A_{J}\right) \lambda\left(A_{J^{c}}\right) \leq\langle\rho(G), \lambda(G)\rangle \leq \operatorname{Hol}(G),
$$

completing the proof of the lemma. 
Lemma 3. The inversion map inv conjugates $G_{J}$ to $G_{J}$ for every $J \subseteq I$.

Proof. The lemma is an immediate consequence of Proposition 1 (3).

Lemma 4. Assume that $G$ is a finite semisimple group. Then, with the above notation, $\mathcal{g}(G)=\left\{G_{J} \mid J \subseteq I\right\}$.

Proof. We first show that $G_{J}$ acts regularly on the set $G$. For an arbitrary $g \in G$, write $g=x_{J} x_{J^{c}}$, with $x_{J} \in A_{J}$ and $x_{J^{c}} \in A_{J^{c}}$. Then the element $\rho\left(x_{J}\right) \lambda\left(x_{J^{c}}^{-1}\right)$ of $G_{J}$ sends 1 to $g$,

$$
1^{\rho\left(x_{J}\right) \lambda\left(x_{J^{c}}\right)}=x_{J^{c}} x_{J}=x_{J} x_{J^{c}}=g,
$$

since $\left[A_{J}, A_{J^{c}}\right]=1$. Moreover, the stabilizer of 1 in $G_{J}$ consists of the elements $\sigma=\rho\left(x_{J}\right) \lambda\left(x_{J^{c}}\right)$ such that $x_{J}=x_{J^{c}}^{-1} \in A_{J} \cap A_{J^{c}} \leq Z(G)$, and therefore $\sigma$ is the identity.

To show that each $G_{J}$ is normal in $\operatorname{Hol}(G)$, since $[\rho(G), \lambda(G)]=1$, it is enough to show that $\operatorname{Aut}(G)$ normalizes every $G_{J}$. Fix $J \subseteq I$; let $\alpha \in \operatorname{Aut}(G)$, and let $\rho\left(x_{J}\right) \lambda\left(x_{J^{c}}\right)$ be an arbitrary element of $G_{J}$, where $x_{J} \in A_{J}$ and $x_{J^{c}} \in A_{J^{c}}$. Then we have that, for every $g \in G$,

$$
g^{\alpha^{-1} \rho\left(x_{J}\right) \lambda\left(x_{J^{c}}\right) \alpha}=\left(x_{J^{c}} g^{\alpha^{-1}} x_{J}\right)^{\alpha}=x_{J^{c}}^{\alpha} g x_{J}^{\alpha}=g^{\rho\left(x_{J}^{\alpha}\right) \lambda\left(x_{J}^{\alpha}\right)} .
$$

Therefore,

$$
\left(\rho\left(x_{J}\right) \lambda\left(x_{J^{c}}\right)\right)^{\alpha}=\rho\left(x_{J}^{\alpha}\right) \lambda\left(x_{J^{c}}^{\alpha}\right),
$$

which lies in $G_{J}$, since $A_{J}$ and $A_{J^{c}}$ are characteristic subgroups of $G$.

Finally, by [5, Theorem 7.8], $|\mathcal{f}(G)|=2^{n}$, and therefore, to complete the proof, it remains to show that $G_{J} \neq G_{K}$ whenever $J \neq K$. If $G_{J}=G_{K}$, then there exists an $i \in I$ for which $G_{J}$ contains both $\rho\left(A_{i}\right)$ and $\lambda\left(A_{i}\right)$. But then the stabilizer of 1 in $G_{J}$ would contain $\left\{\rho(x) \lambda\left(x^{-1}\right) \mid x \in A_{i}\right\}$, that is, all the conjugates of elements of $A_{i}$. Since $A_{i}$ is not central in $G$, this contradicts the fact that $G_{J}$ is regular.

\section{Automorphisms of finite quasisimple groups}

In this section, we classify all finite quasisimple groups that admit an automorphism acting like inversion on the center. This classification, which is used in the proof of our main result, is proved in Proposition 2 using the Classification of Finite Simple Groups. This result completely answers a question posed in [5, Remark 7.12] (see also [2, ADV - 4B]), namely, whether there are finite quasisimple groups $L$ such that $Z(L)$ is not elementary abelian, and such that $\operatorname{Aut}(L)$ does not induce inversion on $Z(L)$, or acts trivially on it. 
Before stating and proving Proposition 2, we introduce some notation and terminology related to automorphisms of finite nonabelian simple groups of Lie type. We refer the interested reader to the first two chapters of [7].

Let $S$ be any finite group of Lie type. Then, by [13, Theorem 30], any automorphism of $S$ is a product $i d f g$, where $i$ is an inner automorphism of $S, d$ is a diagonal automorphism of $S, f$ is a field automorphism of $S$ and $g$ is a graph automorphism of $S$. Using the notation of [7], $\operatorname{Inndiag}(S), \Phi_{S}$ and $\Gamma_{S}$ denote, respectively, the group of inner-diagonal automorphisms of $S$, of field automorphisms of $S$, and of graph automorphisms of $S$. Further, $\operatorname{Outdiag}(S)$ is defined to be $\operatorname{Inndiag}(S) / \operatorname{Inn}(S)$ (see [7, Definition 2.5.10]).

Proposition 2. Let $K$ be a finite quasisimple group. Then there exists an automorphism of $K$ that inverts $Z(K)$ if and only if $K$ is not isomorphic to one of the following groups:

(1) a covering of $\mathrm{L}_{3}(4)$, with center containing $Z_{2} \times Z_{2} \times Z_{3}$,

(2) a covering of $\mathrm{U}_{4}(3)$, with center containing $Z_{3} \times Z_{4}$,

(3) $\mathrm{U}_{6}(2)$, the universal covering of $\mathrm{U}_{6}(2)$,

(4) $\widetilde{{ }^{2} \mathrm{E}_{6}(2)}$, the universal covering of ${ }^{2} \mathrm{E}_{6}(2)$.

Proof. As is well known (see for example [1]), any finite quasisimple group $K$ is isomorphic to a quotient of the universal covering group of its simple quotient $K / Z(K)$. Also, $\operatorname{Aut}(K) \simeq \operatorname{Aut}(K / Z(K))$ (see for instance [1, Section 33]). Therefore, for our purposes, it is enough to consider the action of $\operatorname{Aut}(S)$ on the Schur multiplier $M(S)$ when $S$ varies among all finite nonabelian simple groups. In this situation, the outer automorphism group $\operatorname{Out}(S)$ acts on $M(S)$, which, by [6, Section 5, 6-1], is isomorphic to the direct product of two factors of relatively prime orders, $M_{c}(S)$ and $M_{e}(S)$. The actions of $\operatorname{Out}(S)$ on both factors are completely described in [7, Theorem 6.3.1 and Theorem 2.5.12] for every finite nonabelian simple group $S$. In particular, $\operatorname{Outdiag}(S)$ centralizes $M_{c}(S)$, and there is an isomorphism of $\operatorname{Outdiag}(S)$ on $M_{c}(S)$ preserving the action of $\operatorname{Out}(S)$. Note also that if one of the factors $M_{c}(S)$ or $M_{e}(S)$ has order at most 2, since they have coprime orders, to prove our statement, it is enough to see if inversion is induced by $\operatorname{Out}(S)$ on the other factor. We may therefore consider the two cases:

(1) $\left|M_{e}(S)\right| \leq 2$,

(2) $\left|M_{e}(S)\right|>2$.

Case (1) $\left|M_{e}(S)\right| \leq 2$. We prove that, in this case, there is always an automorphism of $S$ inverting $M(S)$. As noted above, it is enough to consider the action of 
$\operatorname{Out}(S)$ on Outdiag $(S)$, which is $\operatorname{Aut}(S)$-isomorphic to $M_{c}(S)$. But Outdiag $(S)$ is always inverted by $\operatorname{Out}(S)$ since, by [7, Theorem 2.5.12], we have that,

(i) if $S \in\left\{A_{m}(q), D_{2 m+1}(q), \mathrm{E}_{6}(q)\right\}$, then $\operatorname{Outdiag}(S)$ is inverted by a graph automorphism (by [7, Theorem 2.5.12(i)]),

(ii) if $S \in\left\{{ }^{2} A_{m}(q),{ }^{2} D_{2 m+1}(q),{ }^{2} \mathrm{E}_{6}(q)\right\}$, Outdiag $(S)$ is inverted by a field automorphism (by [7, Theorem 2.5.12(g)]),

(iii) in all other cases, $\operatorname{Outdiag}(S)$ is either trivial or an elementary abelian 2group, and therefore it is inverted by the trivial automorphism.

Case (2) $\left|M_{e}(S)\right|>2$. From [7, Table 6.3.1] and the knowledge of the corresponding factor $M_{c}(S)$ ([7, Theorem 2.5.12]), we can see that if $S$ is not isomorphic to one of the simple groups

$$
\mathrm{L}_{3}(4), \mathrm{U}_{6}(2),{ }^{2} \mathrm{E}_{6}(2), \mathrm{U}_{4}(3),
$$

then there exists an automorphism of $S$ that inverts $M(S)$ and, therefore, any quasisimple group $K$ such that $K / Z(K) \simeq S$ admits an automorphism inverting its center. We now consider separately the four special cases listed above.

Let $S=\mathrm{L}_{3}(4)$. Then $M_{e}(S) \simeq Z_{4} \times Z_{4}$ and $M_{c}(S) \simeq Z_{3}$. Here

$$
\operatorname{Out}(S)=\Sigma \times\langle u\rangle,
$$

with $\Sigma=\operatorname{Outdiag}(S) \Gamma_{S} \simeq S_{3}$ and $u$ the image in $\operatorname{Out}(S)$ of a graph-field automorphism of order 2. By [7, Theorem 6.3.1], $u$ is the only element of $\operatorname{Out}(S)$ that induces inversion of $M_{e}(S)$. Now, $u$ is $\operatorname{Aut}(S)$-conjugate to an element of the form $\phi i$, with $\phi$ a field automorphism and $i$ a graph automorphism, where $\phi$ and $i$ are commuting involutions (note that $\Phi_{S} \Gamma_{S} \simeq Z_{2} \times Z_{2}$ ). The action of $\Phi_{S} \Gamma_{S}$ on $M_{c}(S)$ is the same as on $\operatorname{Outdiag}(S)$. Thus, by [7, Theorem 2.5.12(g) and (i)], both $\phi$ and $i$ invert $M_{c}(S)$, and therefore $u$ acts trivially on it. This argument shows that, when $K$ is the universal covering group of $S$, no inversion on $Z(K)$ is induced by an automorphism of $K$. Assume now that $K$ is a covering of $S$ different from the universal one. If $3 \nmid|Z(K)|$, then $u$ inverts $Z(K)$. Assume therefore that 3||$Z(K) \mid$. If $Z(K)$ is cyclic of order 3 or 6 , then $\phi$ inverts $Z(K)$. Otherwise, we may argue as follows. Since $M_{c}(S)$ and $\operatorname{Outdiag}(S)$ are $\operatorname{Aut}(S)$-isomorphic, the elements of $\operatorname{Out}(S)$ that induce inversion on $M_{c}(S)$ are the six non-central involutions, that is, the elements of the set

$$
T=\operatorname{Outdiag}(S) \phi \cup \operatorname{Outdiag}(S) i .
$$

Note that, from [7, Proposition 6.2.2 and the proof of Theorem 6.3.1], Outdiag $(S)$ acts faithfully on the quotient group $M_{e}(S) / \Phi\left(M_{e}(S)\right)$, and hence on $M_{e}(S)$. 
Next, let $t$ be an element of $T$. Since $t$ inverts $\operatorname{Outdiag}(S)$,

$$
C_{M_{e}(S) / \Phi\left(M_{e}(S)\right)}(t) \simeq C_{\Phi\left(M_{e}(S)\right)}(t) \neq \Phi\left(M_{e}(S)\right),
$$

so $C_{M_{e}(S)}(t)=\langle b\rangle \simeq Z_{4}$, and $t$ inverts a unique cyclic subgroup of order 4 . This in particular shows that, when $K$ is a covering extension with

$$
Z(K) \simeq Z_{2} \times Z_{2} \times Z_{3} \text { or } Z(K) \simeq Z_{2} \times Z_{4} \times Z_{3},
$$

no inversion is induced by automorphisms of $K$ on $Z(K)$, while inversion is induced if $Z(K) \simeq Z_{4} \times Z_{3}$.

Let $S=\mathrm{U}_{4}(3)$. Then $M_{e}(S) \simeq Z_{3} \times Z_{3}$ and $M_{c}(S) \simeq Z_{4}$. Here

$$
\operatorname{Out}(S)=\operatorname{Outdiag}(S) \Phi_{S}
$$

is isomorphic to a dihedral group of order 8 acting faithfully on $M_{e}(S)$. In particular, the nontrivial central element of $\operatorname{Out}(S)$ is the unique element inducing inversion on $M_{e}(S)$. Note that this element belongs to $\operatorname{Outdiag}(S)$, and therefore it centralizes $M_{c}(S)$. This implies that no inversion can be induced on $M(S)$ by automorphisms of $S$. Therefore, the universal covering group $\widetilde{S}$ has no automorphisms inverting its center. This argument can be extended to show that the same situation occurs in any covering having center of order 12. However, for all other coverings $\widehat{S}$ of $S$, it can be easily checked that inversion on the center is induced either by the nontrivial central element of $\operatorname{Outdiag}(S) \Phi_{S}$ when 3 divides $|Z(\widehat{S})|$, or by a field automorphism of order two when $3 \nmid|Z(\widehat{S})|$.

Let $S=\mathrm{U}_{6}(2)$, or $S={ }^{2} \mathrm{E}_{6}(2)$. In both cases, we have that

$$
M_{e}(S) \simeq Z_{2} \times Z_{2}, \quad M_{c}(S) \simeq Z_{3},
$$

and $\operatorname{Out}(S) \simeq S_{3}$ acts faithfully on $M_{e}(S)$ (see [7, Proposition 6.2.2]). In particular, the trivial outer automorphism is the only one that inverts $M_{e}(S)$. Since it does not invert $M_{c}(S)$, the universal covering groups $\widetilde{S}$ have no automorphisms inverting their centers. On the contrary, every covering $\widehat{S}$ different from the universal one possesses such automorphisms, which are either trivial if $3 \nmid|Z(\widehat{S})|$, or are field automorphisms.

For convenience, we write $\mathscr{L}$ for the set of quasisimple groups that appear as exceptions in Proposition 2; thus

$$
\begin{aligned}
& \mathscr{L}=\left\{\widehat{\mathrm{L}_{3}(4)}\left(\text { with } Z\left(\widehat{\left(\mathrm{L}_{3}(4)\right.}\right) \geq Z_{2} \times Z_{2} \times Z_{3}\right),\right. \\
& \left.\left.\widehat{\mathrm{U}_{4}(3)} \text { (with } Z\left(\widehat{\mathrm{U}_{4}(3)}\right) \geq Z_{3} \times Z_{4}\right), \widehat{\mathrm{U}_{6}(2)}, \widehat{{ }^{2} \mathrm{E}_{6}(2)}\right\} \text {. }
\end{aligned}
$$


Remark 2. According to [7, Theorem 6.3.2], when $S \simeq \mathrm{L}_{3}$ (4) (or $S \simeq \mathrm{U}_{4}(3)$ ), there are precisely two non-isomorphic covering groups $\widehat{S}$ such that $\widehat{S} / Z(\widehat{S}) \simeq S$ and $Z(\widehat{S}) \simeq Z_{2} \times Z_{4} \times Z_{3}$ (respectively, $\left.Z(\widehat{S}) \simeq Z_{3} \times Z_{4}\right)$. In all other cases in the list $\mathscr{L}$, the covering group of the associated finite simple group is unique. Therefore, up to isomorphism, $|\mathscr{L}|=9$.

As an application of Proposition 2, we prove the following.

Corollary 1. Assume that $X$ is a finite semisimple group with all components not in $\mathscr{L}$. Then there exists an automorphism of $X$ that inverts the center $Z(X)$.

Proof. Let $X$ be a finite semisimple group. Then $X$ is isomorphic to $D / N$, with $D=K_{1} \times K_{2} \times \cdots \times K_{t}$ the direct product of nonabelian quasisimple groups $K_{i} \notin \mathscr{L}$ and $N \leq D$ such that $N \cap K_{i}=1$ for each $i \in\{1, \ldots, t\}$. Note that

$$
N \leq Z(D)=Z\left(K_{1}\right) \times Z\left(K_{2}\right) \times \cdots \times Z\left(K_{t}\right) .
$$

By Proposition 2, for each $i \in\{1, \ldots, t\}$ there exists an automorphism $\alpha_{i}$ of $K_{i}$ that acts like the inversion on $Z\left(K_{i}\right)$. We may therefore define $\alpha \in \operatorname{Aut}(D)$ by $x^{\alpha}=\left(x_{1}, x_{2}, \ldots, x_{t}\right)^{\alpha}=\left(x_{1}^{\alpha_{1}}, x_{2}^{\alpha_{2}}, \ldots, x_{t}^{\alpha_{t}}\right)$ for $x \in D$ (where each $\left.x_{i} \in K_{i}\right)$. Note that every element of $Z(D)$ is inverted by $\alpha$. In particular, $N^{\alpha}=N$, and $\alpha$ induces an automorphism on $D / N$ that inverts its center.

\section{The holomorph of a semisimple group}

We first consider the case in which $G$ has no components belonging to $\mathscr{L}$.

Proposition 3. Assume that $G$ is a finite nonabelian semisimple group, and let $G=A_{1} A_{2} \ldots A_{n}$ be its unique central decomposition as a product of $\operatorname{Aut}(G)$ indecomposable factors. Suppose that the components of $G$ do not belong to $\mathscr{L}$. Then $\mathscr{H}(G)=\left\{G_{J} \mid J \subseteq I\right\}$. Moreover, the group $T(G)=\operatorname{NHol}(G) / \operatorname{Hol}(G)$ is elementary abelian of order $2^{n}$.

Proof. By Lemma 4, we have $\mathcal{A}(G)=\left\{G_{J} \mid J \subseteq I\right\}$, so $\mathscr{H}(G) \subseteq\left\{G_{J} \mid J \subseteq I\right\}$. We fix a subset $J$ of $I$ and, using Corollary 1 , choose an automorphism $\alpha_{J^{c}}$ of $A_{J^{c}}$ that inverts $Z\left(A_{J^{c}}\right)$. Define the map $\varphi_{J}: G \rightarrow G$ by $\varphi_{J}\left(x_{J} x_{J^{c}}\right)=x_{J}\left(x_{J^{c}}\right)^{-\alpha_{J c}}$. Since $\alpha_{J^{c}}$ inverts $A_{J} \cap A_{J^{c}} \leq Z(G)$, the map $\varphi_{J}$ is a well-defined bijection of $G$, that is, an element of $S(G)$. We claim that the following hold:

(1) $\varphi_{J}$ conjugates $G_{I}$ to $G_{J}$;

(2) $\varphi_{J} \in \operatorname{NHol}(G)$ and, if $J \neq I$, then $\varphi_{J} \notin \operatorname{Hol}(G)$;

(3) $\left(\varphi_{J}\right)^{2} \in \operatorname{Hol}(G)$. 
Note that, by the arbitrary choice of $J \subseteq I$, once proved, (1) will imply that $\mathscr{H}(G)=\mathscr{H}(G)=\left\{G_{J} \mid J \subseteq I\right\}$, while (2), (3) and the fact that $T(G)$ acts regularly on $\mathscr{H}(G)$ will imply that $T(G)$ is an elementary abelian 2-group of rank $n$.

(1) $\left(G_{I}\right)^{\varphi_{J}}=G_{J}$. We claim that, for $x_{J} \in A_{J}$ and $x_{J^{c}} \in A_{J^{c}}$,

$$
\left(\rho\left(x_{J} x_{J^{c}}\right)\right)^{\varphi_{J}}=\rho\left(x_{J}\right) \lambda\left(x_{J^{c}}^{-\alpha_{J^{c}}}\right)
$$

or, equivalently, that

$$
\rho\left(x_{J} x_{J^{c}}\right) \cdot \varphi_{J}=\varphi_{J} \cdot \rho\left(x_{J}\right) \lambda\left(x_{J^{c}}^{-\alpha_{J^{c}}}\right) .
$$

Let $g \in G$, and write $g$ as $g=y_{J} y_{J^{c}}$ (with $y_{J} \in A_{J}$ and $y_{J^{c}} \in A_{J^{c}}$ ). Then

$$
\begin{aligned}
g^{\rho\left(x_{J} x_{J c}\right) \cdot \varphi_{J}} & =\left(y_{J} y_{J^{c}} x_{J} x_{J^{c}}\right)^{\varphi_{J}}=\left(y_{J} x_{J} y_{J^{c}} x_{J^{c}}\right)^{\varphi_{J}} \\
& =y_{J} x_{J}\left(y_{J^{c}} x_{J^{c}}\right)^{-\alpha_{J^{c}}}=y_{J} x_{J} x_{J^{c}}^{-\alpha_{J^{c}}} y_{J^{c}}^{-\alpha_{J^{c}}}, \\
g^{\varphi_{J} \cdot \rho\left(x_{J}\right) \lambda\left(x_{J^{c}}^{-\alpha_{J^{c}}}\right)} & =\left(y_{J} y_{J^{c}}^{-\alpha_{J^{c}}}\right)^{\rho\left(x_{J}\right) \lambda\left(x_{J^{c}}^{-\alpha_{J^{c}}}\right)}=x_{J^{c}}^{-\alpha_{J^{c}}} y_{J} y_{J^{c}}^{-\alpha_{J^{c}}} x_{J} \\
& =y_{J} x_{J} x_{J^{c}}^{-\alpha_{J^{c}}} y_{J^{c}}^{-\alpha_{J^{c}}} .
\end{aligned}
$$

Therefore, (1) is proved. Note that, together with Lemma 4, we have proved that $\mathscr{L}(G) \subseteq \mathscr{H}(G)$ and therefore $\mathscr{H}(G)=\mathscr{l}(G)=\mathscr{L}(G)$.

(2) $\varphi_{J} \in \operatorname{NHol}(G)$. By (1), we have that

$$
\left(N_{S(G)}\left(G_{I}\right)\right)^{\varphi_{J}}=N_{S(G)}\left(G_{I}^{\varphi_{J}}\right)=N_{S(G)}\left(G_{J}\right)=N_{S(G)}\left(G_{I}\right)
$$

since each $G_{J}$ lies in $\mathscr{H}(G)=\mathcal{l}(G)$, and therefore $N_{S(G)}\left(G_{J}\right)=N_{S(G)}\left(G_{I}\right)$; thus $\varphi_{J} \in \operatorname{NHol}(G)$.

Furthermore, $G_{J} \neq G_{I}$ for $J \neq I$; thus we trivially have that $\varphi_{J} \notin \operatorname{Hol}(G)$ for $J \neq I$.

(3) $\left(\varphi_{J}\right)^{2} \in \operatorname{Hol}(G)$. We claim that, for every $x_{J} x_{J^{c}} \in G$,

$$
\left(\rho\left(x_{J} x_{J^{c}}\right)\right)^{\varphi_{J}^{2}}=\rho\left(x_{J} x_{J^{c}}^{\alpha^{2}}\right)
$$

or, equivalently, that

$$
\rho\left(x_{J} x_{J^{c}}\right) \cdot \varphi_{J}^{2}=\varphi_{J}^{2} \cdot \rho\left(x_{J} x_{J^{c}}^{\alpha^{c}}\right) .
$$

Let $g \in G$, and write $g=y_{J} y_{J^{c}}$, where $y_{J} \in A_{J}, y_{J^{c}} \in A_{J^{c}}$. Then

$$
\begin{aligned}
g^{\rho\left(x_{J} x_{J c}\right) \cdot \varphi_{J}^{2}} & =\left(y_{J} y_{J^{c}} x_{J} x_{J^{c}}\right)^{\varphi_{J}^{2}}=\left(y_{J} x_{J} y_{J^{c}} x_{J^{c}}\right)^{\varphi_{J}^{2}} \\
& =y_{J} x_{J}\left(y_{J^{c}} x_{J^{c}}\right)^{\alpha^{2}}=y_{J} y_{J^{c}}^{\alpha^{c}} x_{J} x_{J^{c}}^{\alpha^{c}},
\end{aligned}
$$


while

$$
g^{\varphi_{J}^{2} \cdot \rho\left(x_{J} x_{J^{c}}^{\alpha^{c}}\right)}=\left(y_{J} y_{J^{c}}^{\alpha^{c}}\right)^{\rho\left(x_{J} x_{J^{c}}^{\alpha^{c}}\right)}=y_{J} y_{J^{c}}^{\alpha^{c}} x_{J} x_{J^{c}}^{\alpha^{c}}
$$

This completes the proof of Proposition 3.

Remark 3. For each fixed subset $J$ of $I$, we may define an operation $\circ_{J}$ on the set of elements of $G$ as follows:

$$
g \circ_{J} h=g_{J} g_{J} \circ_{J} h_{J} h_{J c}=g_{J} h_{J} h_{J} g_{J^{c}}
$$

for each $g=g_{J} g_{J^{c}}, h=h_{J} h_{J^{c}} \in G$, where $g_{J}, h_{J} \in A_{J}$ and $g_{J^{c}}, h_{J^{c}} \in A_{J^{c}}$. Then $\left(G, \circ_{J}\right)$ is a group. Note that $\circ_{I}$ coincides with the group operation of $G$, while $\circ \varnothing$ with the opposite multiplication in $G$, that is, $g_{1} \circ \varnothing g_{2}=g_{2} g_{1}$ for every $g_{1}, g_{2} \in G$. With this notation, it is straightforward to prove that

(1) $\left(G, \circ_{J}\right)$ is a group isomorphic to $G_{J}$ for each $J \subseteq I$,

(2) $\operatorname{Aut}(G)=\operatorname{Aut}\left(G, \circ_{J}\right)$ for each $J \subseteq I$ (see [5, Theorem 5.2(d)],

(3) if $G$ satisfies the assumptions of Proposition 3, each map $\varphi_{J}$ is an isomorphism between $\left(G, \circ_{I}\right)$ and $\left(G, \circ_{J}\right)$.

We consider now the general situation in which exactly $l$ components of $G$ do belong to $\mathscr{L}$. If $K \in \mathscr{L}$, we call $\mathscr{L}$-critical any subgroup $U$ of $Z(K)$ such that, respectively,

- $U \simeq Z_{2} \times Z_{2} \times Z_{3}$ if $K \simeq \widehat{\mathrm{L}_{3}(4)}$,

- $U \simeq Z_{3} \times Z_{4}$ if $K \simeq \widehat{\mathrm{U}_{4}(3)}$,

- $U=Z(K)$ if $K \simeq \widetilde{\mathrm{U}_{6}(2)}$ or if $K=\widetilde{{ }^{2} \mathrm{E}_{6}(2)}$.

Theorem 1. Let $G$ be a finite nonabelian semisimple group, and let

$$
G=A_{1} A_{2} \ldots A_{n}
$$

be its unique central decomposition as a product of Aut $(G)$-indecomposable factors. Assume that the number of factors $A_{i}$ of $G$ having components in $\mathscr{L}$ is exactly $l$ for some $0 \leq l \leq n$. Then $T(G)$ is an elementary abelian group of order $2^{h}$ for some $h$ with $\min \{n-l+1, n\} \leq h \leq n$, acting regularly on $\mathscr{H}(G)$. Moreover, if the centers of the factors $A_{i}$ are all amalgamated, then $|\mathcal{H}(G)|=2^{m}$, and $T(G)$ is elementary abelian of order $2^{m}$, where $m=\min \{n, n-l+1\}$, and therefore $|\mathscr{H}(G)|=2^{m}$. 
Proof. By Proposition 3, the result is clear for $l=0$, so assume $l>0$.

Without loss of generality, we may assume that $A_{1}, A_{2}, \ldots, A_{l}$ are the central $\operatorname{Aut}(G)$-indecomposable factors having components in $\mathscr{L}$. We set $L=\{1,2, \ldots, l\}$ and claim that $\mathscr{H}(G)$ contains the set $\mathcal{K}=\left\{G_{J}, G_{J^{c}} \mid J \cap L=\varnothing\right\}$, whose cardinality is $2 \cdot\left|\mathcal{P}\left(L^{c}\right)\right|=2^{m}$. By the definition of $\mathscr{H}(G)$ and Lemmas 3 and 2, it is enough to show that $G_{J^{c}} \simeq G_{I}$ for each subset $J$ of $L^{c}$. By Corollary 1, there exists an automorphism $\alpha_{J}$ of $A_{J}$ that inverts $Z\left(A_{J}\right)$. Therefore, the map $\varphi_{J} c$ defined as in Proposition 3 by $\varphi_{J} c\left(x_{J} x_{J^{c}}\right)=\left(x_{J}\right)^{-\alpha_{J}} x_{J^{c}}$ for each $x_{J} \in A_{J}$, $x_{J^{c}} \in A_{J^{c}}$ is a well-defined bijection of $G$ that conjugates $G_{I}$ to $G_{J^{c}}$. Note that $T(G)$ contains the elementary abelian 2-subgroup $\left\{\varphi_{J^{c}} \mid J \cap L=\varnothing\right\}$.

Assume now that $\mathcal{K} \subset \mathscr{H}(G)$. Note that $G_{R} \in \mathscr{H}(G) \backslash \mathcal{K}$ for some $R \subset I$ if and only if $G_{R^{c}} \in \mathscr{H}(G) \backslash \mathcal{K}$. This shows in particular that $|\mathcal{H}(G)|$ is even.

Moreover, by Remark 3, $G_{R} \in \mathscr{H}(G) \backslash \mathcal{K}$ if and only if $G_{R}$ is isomorphic to the group $\left(G, \circ_{R}\right)$. Now, by Remark 3, any possible isomorphism $\alpha$ from $G$ to $\left(G, \circ_{R}\right)$ maps each $A_{i}$ to itself. In particular, if we take $r \in R \cap L, s \in R^{c} \cap L$, the isomorphism $\alpha$ induces an automorphism on $A_{r}$ and an antihomomorphism on $A_{s}$, as we have

$$
\begin{array}{ll}
\left(a_{r} b_{r}\right)^{\alpha}=a_{r}^{\alpha} b_{r}^{\alpha}=a_{r}^{\alpha} \circ_{J} b_{r}^{\alpha} & \text { for each } a_{r}, b_{r} \in A_{r}, \\
\left(a_{s} b_{s}\right)^{\alpha}=a_{s}^{\alpha} b_{s}^{\alpha}=b_{s}^{\alpha} \circ_{J} a_{s}^{\alpha} & \text { for each } a_{s}, b_{s} \in A_{s} .
\end{array}
$$

Condition (4.1) implies that the restriction of $\alpha$ to $W=A_{r} \cap A_{s}$ is a homomorphism, while condition (4.2) implies that the restriction of $\alpha \circ$ inv to $W$ is a homomorphism. We deduce that the inversion map on $W$ is induced by an automorphism of $A_{r}$, which is in contradiction with Proposition 2 if $W$ contains an $\mathscr{L}$-critical subgroup for some component of $A_{r}$. Thus we have proved that $G_{R} \in \mathscr{H}(G) \backslash \mathcal{K}$ if and only if, for every $r \in R \cap L$ and every $s \in R^{c} \cap L$, the subgroup $A_{r} \cap A_{s}$ does not contain $\mathscr{L}$-critical subgroups of components. Note that this is equivalent to saying that the involutory map $\varphi_{R}$ is an element of $T(G)$. In particular, $T(G)$ is an elementary abelian 2-group of order $|\mathscr{H}(G)|$, which is therefore a power of 2.

When $Z\left(A_{i}\right)=Z(G)$ for each $i=1,2, \ldots, n$, the result is clear since each $A_{r} \cap A_{s}=Z(G)$.

\section{Bibliography}

[1] M. Aschbacher, Finite Group Theory, 2nd ed., Cambridge University, Cambridge, 2000 .

[2] A. Caranti, ADV perspectives in group theory, Adv. Group Theory Appl. 4 (2017), 131-138. 
[3] A. Caranti, Multiple holomorphs of finite p-groups of class two, J. Algebra 516 (2018), 352-372.

[4] A. Caranti and F. Dalla Volta, The multiple holomorph of a finitely generated abelian group, J. Algebra 481 (2017), 327-347.

[5] A. Caranti and F. Dalla Volta, Groups that have the same holomorph as a finite perfect group, J. Algebra 507 (2018), 81-102.

[6] D. Gorenstein and R. Lyons, The local structure of finite groups of characteristic 2 type, Mem. Amer. Math. Soc. 42 (1983), no. 276, 1-731.

[7] D. Gorenstein, R. Lyons and R. Solomon, The Classification of the Finite Simple Groups. Number 3. Part I. Chapter A, Math. Surveys Monogr. 40, American Mathematical Society, Providence, 1998.

[8] N. J. S. Hughes, The structure and order of the group of central automorphisms of a finite group, Proc. Lond. Math. Soc. (2) 52 (1951), 377-385.

[9] T. Kohl, Multiple holomorphs of dihedral and quaternionic groups, Comm. Algebra 43 (2015), no. 10, 4290-4304.

[10] G. A. Miller, On the multiple holomorphs of a group, Math. Ann. 66 (1908), no. 1, 133-142.

[11] W. H. Mills, Multiple holomorphs of finitely generated abelian groups, Trans. Amer. Math. Soc. 71 (1951), 379-392.

[12] D. J. S. Robinson, A Course in the Theory of Groups, 2nd ed., Grad. Texts in Math. 80, Springer, New York, 1996.

[13] R. Steinberg, Lectures on Chevalley Groups, Yale University, New Haven, 1968.

Received January 28, 2020; revised August 25, 2020.

\section{Author information}

Russell D. Blyth, Department of Mathematics and Statistics, Saint Louis University, 220 N. Grand Blvd., St. Louis, MO 63103, USA.

E-mail: russell.blyth@slu.edu

Corresponding author:

Francesco Fumagalli, Dipartimento di Matematica e Informatica "Ulisse Dini",

Università degli Studi di Firenze, viale Morgagni 67/A, 50134 Firenze, Italy.

E-mail: francesco.fumagalli@unifi.it 